\section{Complementary}

Medicine Research

Practice $\mid$ Methods $\mid$ Perspectives
Published online: June 21, 2018

\title{
Natur, Naturheilkunde, Naturwissenschaft: Vom Monte Verità zur Molekularmedizin
}

\author{
Andreas Michalsen ${ }^{a, b}$ \\ ${ }^{a}$ Institut für Sozialmedizin, Epidemiologie und Gesundheitsökonomie, \\ Charité - Universitätsmedizin Berlin, Berlin, Deutschland; \\ bZentrum für Naturheilkunde, Immanuel Krankenhaus Berlin, Berlin, Deutschland
}

Die Geschichte der Naturheilkunde ist geprägt von Ärzten und Laientherapeuten, die oft aus eigener Heilungserfahrung und reformerischen idealistischen Impulsen die Nähe zur Natur suchten und natürliche therapeutische Reize, oftmals intuitiv, mit großem Erfolg als Therapieprinzipien entwickelten. In Bad Wörishofen setzte Sebastian Kneipp Wasser, Heilpflanzen und Barfußlaufen ein, der «Sonnendoktor» Arnold Rikli verordnete Licht- und Luftbäder, Max Bircher-Benner propagierte die intensive Ernährungstherapie und Otto Buchinger das Fasten. Eingebettet in die Sehnsucht sozialer Utopien kulminierte dieses naturromantische und reformerische Denken zu Beginn des 20. Jahrhunderts in der Künstlerkolonie und Naturheilanstalt am Tessiner Monte Verità. Hier trafen sich Hermann Hesse, Erich Mühsam, Martin Buber, Rudolf von Laban und viele mehr und suchten neue Lebenswege mit veganer Ernährung, Lichthütten und Ausdruckstanz. Es war die therapeutisch motivierte Abwendung vom Leben in den früh industrialisierten Großstädten mit ihren damals ausgeprägten ökologischen und sozialen Problemen vor dem Hintergrund eines politisch unruhigen Europas. Die Vorstellung eines gesunden und inspirierenden naturnahen Lebens ließ sie die Reise nach Ascona antreten.

Die industrielle Produktion und die Lebensbedingungen in unserer Gesellschaft haben sich seither dramatisch gewandelt. Rauchende Fabrikschlote und dunkle Berliner Hinterhöfe mit prekären Arbeitsbedingungen sind «Smart Technologies», der Digitalisierung, der Dienstleistung und einer neuen, modernen Urbanisierung gewichen. Trotz der Vorteile des modernen urbanen Lebens zeigt sich an vielen Stellen eine neue drängende Sehnsucht nach der Natur. Bücher über den Wald, die Natur oder die Tierwelt haben politische Themen von den Bestsellerlisten verdrängt. Paläo-Diäten und Intervallfasten faszinieren die Menschen durch die Aussicht, so mehr im Einklang mit der gegebenen körperlichen Biologie zu leben. Tautreten und die Kneipp-Sandale finden ihre moderne Entsprechung in teuren Barfußschuhen und «Earthing». Doch während die frühe Naturheilkunde vor allem ein Gegenentwurf zur herrschenden Schulmedizin und eine ausschließliche Erfahrungs- und intuitive Medizin war, scheint nun eine Reunion von Naturheilkunde, Ökologie und Naturwissenschaft stattzufinden.

Vor allem die Molekularbiologie und Epigenetik zeigen inzwischen ein reges Interesse an natürlichen Interventionen. Der Molekularmediziner und ehemalige Charité-Chef Detlef Ganten definierte die «Evolutionäre Medizin» als geeignete Antwort auf die Epidemie der Zivilisationskrankheiten [1]. So wurden in den letzten Jahren Wirksamkeits-

\section{KARGER}

() 2018 S. Karger GmbH, Freiburg

Fax +497614520714 
«Pathways» des periodischen und intermittierenden Fastens umfänglich identifiziert $[2,3]$, und in der Folge ist die translationale klinische Überprüfung des Fastens für viele neue Indikationen in vollem Gang. Grundlagenwissenschaftliche Arbeiten zur Genexpression von Blutzellen dokumentierten die Temperatur- und Jahreszeitenabhängigkeit der Expression von Schlüsselgenen der Immunfunktion [4]. Forscher wie Satchidananda Panda vom Scripps Institute erforschen das Zusammenspiel von Essen und Fasten sowie Licht und Tages-Nacht-Rhythmik $[5,6]$ und haben erst kürzlich einen ersten Transkriptom-Atlas zu diesem Thema publiziert [7]. Die bedeutende Rolle der Ernährung wird durch den Megatrend der Mikrobiomforschung befeuert; unlängst zeigten die international sehr beachteten Arbeiten von Erin Segal, dass jede einzelne Mahlzeit, ihre Komponenten und auch die Lebensumgebung zu distinkten individuellen Änderungen des Mikrobioms und Metabolismus führen [8,9]. Schon wird sogar spekuliert, ob die probiotische Therapie in Zukunft die zunehmend schwierig gewordene antibiotische Therapie zu ersetzen vermag. Und schon länger beeindruckt die Mind-Body-Medizin durch ihre Vielzahl an neurobiologischen Studien. Selbst die Medizin-Nobel-Preise zeigen auf einmal eine große Nähe zu Themen der Naturheilkunde (Artemisinin 2015, Autophagie 2016, Chronobiologie 2017), und dies reflektiert, dass die systembiologische immer mehr die klassische monohypothetische Sichtweise ablöst.

Auch in der klinischen Forschung spiegelt sich dieses Interesse an der Naturheilkunde. Als Beispiele seien neuere Studien zu den kardioprotektiven Wirkungen der Sauna [10], randomisierte kontrollierte Studien, die die Wirksamkeit von Lichttherapie und Hyperthermie bei Depression belegen [11, 12], oder die wachsende Zahl japanischer Studien zum Thema «forest medicine» und «Shinrin Yoku» (Waldbaden) genannt. Dieses Thema illustriert auch die Multimodalität von Naturmedizin. Während einige Studien die Bewegungskomponente und die Wirkung der aromatischen Öle beim Waldspaziergang herausstellten, zeigte eine der jüngsten Untersuchungen, dass sich selbst bei reiner Betrachtung eines Waldbilds auf einem Plasmabildschirm für $90 \mathrm{~s}$ eine $\mathrm{Zu}$ nahme von Entspannung und Wohlbefinden mit neurobiologischem Korrelat im präfrontalen Cortex einstellte [13]. Auch die Heliotherapie stößt inzwischen wieder auf Interesse. Epidemiologische Studien konnten zeigen, dass Sonnenexposition mit einer Reduktion des Risikos von kardiovaskulären und Krebserkrankungen einhergeht [14], und einige neuere klinische Studien legen den Schluss nahe, dass eine reine Vitamin-D-Supplementierung wahrscheinlich nicht die umfassenderen Effekte des Sonnenlichts ersetzen kann. Abgesehen davon belegt jedes Jahr im Frühling die Alltagsbeobachtung, wie sehr die Menschen die Sonne lieben, die offensichtlich einen stimmungshebenden Effekt hat.

$\mathrm{Zu}$ hoffen bleibt, dass die aufgezeigte Entwicklung auch auf die Phytotherapie übergreift. Aus der Perspektive einer evolutionären Medizin ist es naheliegend, dass ein koentwickeltes pflanzliches Vielstoffgemisch einen stärkeren Zugang zur systembiologischen Steuerung des Körpers hat als eine hochdosierte Single-TargetSubstanz oder ein Rezeptor-Antikörper, die jeweils nur an einer Stelle in einen pathologischen Pathway eingreifen.
Ein letzter wichtiger Aspekt dieser Forschungsentwicklung ist die zunehmende Nutzung von Bioinformatik und Big Data und damit auch eine gewisse Abkehr von der klassischen klinischen Studie mit Mittelwertsvergleichen. Forschung zu Themen wie «Mikrobiom» oder «Epigenetik» ist per se stärker personalisiert und öffnet so ein Fenster für eine neue Individualisierung in der Medizin. Das Zauberwort ist "precision medicine». Hier ist dann die spannende die Frage, ob eine algorithmengestützte Medizin, die sich die «precision medicine» zunutze macht, auch dasselbe Ziel verfolgt wie das von der Erfahrungsmedizin der Naturheilkunde phänomenologisch entwickelte. Es scheint, dass die menschliche Intuition und Erfahrung schon jetzt teilweise nicht mehr mit den Ergebnissen einer Hochdurchsatz- bzw. Systembiologie mithalten können. Aber die Ergebnisse aus solchen Prozessen müssen erst validiert werden. Wird die personalisierte Ernährung von Segals «Personalized Nutrition Project» besser sein als die Erfahrung eines Fasten- oder Mayr-Arztes? So bleibt zunächst fraglich, ob durch die Systembiologie eine auch praktikable Individualisierung der Therapie möglich wird oder ob hier nicht eher der Rückgriff auf die Erfahrung der Naturheilkunde eine reellere Option darstellt. Oder kann es am Ende zu einer Integration von Grundlagenwissenschaft und der Erfahrung der Naturheilkunde kommen? Erste Arbeiten, z.B. von van der Greef et al. [15], haben gezeigt, dass die Systembiologie und diagnostisch-phänomenologische Konzepte der traditionellen chinesischen Medizin tatsächlich zu ähnlichen Resultaten führen können.

Durch die rasanten Entwicklungen in der noch jungen Disziplin «Systembiologie» können systemische Prozesse zweifellos besser erfasst und beschrieben werden. In der Arzneimittelforschung will man mittels der Systembiologie beispielsweise die Dynamik von Medikamentenwirkungen besser verstehen, um somit noch spezifischer zu werden. Das größte Potenzial scheint aber in der Modellierung von holistischen Prozessen, wie sie in der Naturheilkunde vorkommen, zu liegen.

In der Konsequenz entstehen neue große Chancen der wissenschaftlichen Durchdringung für unser Fach. Diese aktuelle Entwicklung wirkt, zumindest aktuell, vielversprechender als große klinische Studien und die damit verbundene perpetuiert mühsame Suche nach Finanzierungsquellen. Letztlich können solche Studien dem Vergleich mit der industriegeförderten konventionellen Forschung kaum mehr Stand halten (Stichwort: «financial bias»). Somit bietet die neue Öffnung der Grundlagenwissenschaft, der translationalen Forschung und der Systembiologie unerwartete neue Möglichkeiten im Hinblick auf Kooperationen mit Impact und Relevanz für die weitere Integration unseres Fachs in die moderne Medizin.

\section{Disclosure Statement}

Es bestehen keine Interessenkonflikte. 


\section{Literatur}

1 Ganten D: Die Steinzeit steckt uns in den Knochen: Gesundheit als Erbe der Evolution. München, Zürich, Piper, 2011.

2 Longo VD, Mattson MP: Fasting: molecular mechanism and clinical applications. Cell Metab 2014;19:181-192.

3 Mattson MP, Longo VD, Harvie M: Impact of intermittent fasting on health and disease processes. Ageing Res Rev 2017;39:46-58.

4 De Jong S, Neeleman M, Luykx JJ, ten Berg MJ, Strengman E, Den Breeijen HH, Stijvers LC, Buizer-Voskamp JE, Bakker SC, Kahn RS, Horvath S, Van Solinge WW Ophoff RA: Seasonal changes in gene expression represent cell-type composition in whole blood. Hum Mol Genet 2014;23:2721-2728.

5 Panda S: Circadian physiology of metabolism. Science 2016;354:1008-1015.

6 Longo VD, Panda S: Fasting, circadian rhythms, and time-restricted feeding in healthy lifespan. Cell Metab 2016;23:1048-1059.

7 Mure LS, Le HD, Benegiamo G, Chang MW, Rios L, Jillani N, Ngotho M, Kariuki T, Dkhissi-Benyahya O, Cooper HM, Panda S: Diurnal transcriptome atlas of a primate across major neural and peripheral tissues. Science 2018;359:pii: eaao0318
Thaiss CA, Levy M, Korem T, Dohnalová L, Shapiro H, Jaitin DA, David E, Winter DR, Gury-BenAri M, Tatirovsky E, Tuganbaev T, Federici S, Zmora N, Zeevi D, Dori-Bachash M, Pevsner-Fischer M, Kartvelishvily E, Brandis A, Harmelin A, Shibolet O, Halpern Z, Honda K, Amit I, Segal E, Elinav E: Microbiota diurnal rhythmicity programs host transcriptome oscillations. Cell 2016;167:1495-1510

9 Rothschild D, Weissbrod O, Barkan E, Kurilshikov A, Korem T, Zeevi D, Costea PI, Godneva A, Kalka IN, Bar N, Shilo S, Lador D, Vila AV, Zmora N, PevsnerFischer M, Israeli D, Kosower N, Malka G, Wolf BC, Avnit-Sagi T, Lotan-Pompan M, Weinberger A, Halpern Z, Carmi S, Fu J, Wijmenga C, Zhernakova A, Elinav E, Segal E: Environment dominates over host genetics in shaping human gut microbiota. Nature 2018; 555:210-215

10 Laukkanen T, Khan H, Zaccardi F, Laukkanen JA: Association between sauna bathing and fatal cardiovascular and all-cause mortality events. JAMA Intern Med 2015;175:542-548.
1 Lam RW, Levitt AJ, Levitan RD, Michalak EE, Cheung AH, Morehouse R, Ramasubbu R, Yatham LN, Tam EM: Efficacy of bright light treatment, fluoxetine, and the combination in patients with nonseasonal major depressive disorder: a randomized clinical trial. JAMA Psychiatry 2016;73:56-63.

12 Janssen CW, Lowry CA, Mehl MR, Allen JJ, Kelly KL, Gartner DE, Medrano A, Begay TK, Rentscher K, White JJ, Fridman A, Roberts LJ, Robbins ML, Hanusch KU,Cole SP, Raison CL: Whole-body hyperthermia for the treatment of major depressive disorder: a randomized clinical trial. JAMA Psychiatry 2016;73: 789-795.

13 Song C, Ikei H, Miyazaki Y: Physiological effects of visual stimulation with forest imagery. Int J Environ Res Public Health 2018;15:pii: E213.

14 Lindqvist PG: The winding path towards an inverse relationship between sun exposure and all-cause mortality. Anticancer Res 2018;38:1173-1178.

5 van der Greef J, van Wietmarschen H, Schroën J, Wang M, Hankemeier T, Xu G: Systems biology-based diagnostic principles as pillars of the bridge between Chinese and Western medicine. Planta Med 2010;76:2036-2047. 